\title{
CONCEPÇÃO DOS PROFISSIONAIS DA ATENÇÃO PSICOSSOCIAL SOBRE INCLUSÃO SOCIAL*
}

Elisângela Braga de Azevedo¹, Rafael Nicolau Carvalho², Renata Cavalcanti Cordeiro³, Camilla de Sena Guerra³, Lawrencita Limeira Espinola ${ }^{4}$, Maria de Oliveira Ferreira Filha ${ }^{5}$

RESUMO: Objetivou-se conhecer a concepção sobre Inclusão Social dos profissionais que atuam na atenção psicossocial de Campina Grande - Paraíba. Trata-se de uma pesquisa descritiva-interpretativa e qualitativa, realizada entre junho e julho de 2010 com 19 profissionais. Para a produção do material empírico utilizou-se entrevista semiestruturada e análise de conteúdo como técnica de análise. Percebeu-se que os profissionais apresentam uma concepção sobre Inclusão Social associada ao paradigma da reabilitação/reinserção demonstrando que os usuários precisam ser resgatados e reinseridos no convívio com a comunidade e com os grupos sociais para serem reconhecidos como cidadãos. Os participantes fazem mensão à inclusão social como uma possibilidade de acesso/acessibilidade nos ambientes sociais imprimindo, em suas falas, a necessidade de inclusão desses em experiências educacionais, lazer e compras, entre outros. Os profissionais cumprem seu papel no contexto da Reforma Psiquiátrica, promovendo saúde mental a partir da inserção social. DESCRITORES: Saúde mental; Serviços de saúde mental; Profissional da saúde.

\section{PSYCHOSOCIAL CARE PROFESSIONALS' CONCEPTIONS ON SOCIAL INCLUSION}

ABSTRACT: This study aimed to investigate the conceptions on Social Inclusion held by professionals who work in psychosocial care in Campina Grande in the state of Paraíba. It is descriptive-interpretive and qualitative research, undertaken between June and July 2010 with 19 professionals. Semi-structured interviews were used for production of the empirical material and content analysis as the technique for analysis. It was perceived that the professionals have a conception of Social Inclusion which is associated with the paradigm of rehabilitation/re-insertion, showing that the service users need to be rescued and re-inserted into co-existence with the community and with the social groups in order to recognized as citizens. The participants refer to social inclusion as a possibility for access/accessibility in social environments, imprinting, in their discourses, the need for inclusion of these in educational experiences, leisure and shopping, among others. The professionals fulfil their role in the context of the Psychiatric Reform, promoting mental health based on social insertion. DESCRIPTORS: Mental health; Mental health services; Health professional.

\section{CONCEPCIÓN DE LOS PROFESIONALES DE LA ATENCIÓN PSICOSOCIAL ACERCA DE LA INCLUSIÓN SOCIAL}

RESUMEN: La finalidad de este estudio fue conocer la concepción sobre Inclusión Social de los profesionales del área de atención psicosocial de Campina Grande, Paraíba. Es una investigación descriptiva, interpretativa y cualitativa, realizada entre junio y julio de 2010 con 19 profesionales. Para la producción del material empírico, fue utilizada la entrevista semiestructurada y el análisis de contenido como técnica de análisis. Se percibió que los profesionales presentan una concepción sobre Inclusión Social asociada al paradigma de rehabilitación/reinserción, dando muestras de que los usuarios necesitan ser rescatados y reinjeridos en la comunidad y en grupos sociales para, de esa manera, se reconocerlos como ciudadanos. Los participantes hablaron de inclusión social como una posibilidad de acceso/accesibilidad a los ambientes sociales, dando muestras de la necesidad de inclusión de eses en experiencias educacionales, ocio y compras, entre otros. Los profesionales cumplen su papel en el contexto de la Reforma Psiquiátrica, promoviendo salud mental a partir de la inserción social. DESCRIPTORES: Salud mental; Servicios de salud mental; Profesional de la salud.

\footnotetext{
* Este artigo é um recorte da dissertação 'Rede de Cuidado em Saúde Mental: Tecendo Práticas de Inclusão Social no município de Campina Grande- PB' apresentada ao Programa de Pós-Graduação em Enfermagem da Universidade Federal da Paraíba - UFPB, em 2010.

${ }^{1}$ Enfermeira. Mestre em Enfermagem. Doutoranda pelo Programa de Pós-Graduação em Enfermagem da UFPB. Professora da Faculdade de Ciências Médicas de Campina Granda. Membro do Grupo de Estudos e Pesquisas em Saúde Mental Comunitária-GEPSMEC.

${ }^{2}$ Assistente Social. Doutorando pelo Programa de Pós-Graduação em Sociologia da UFPB. Coordenador do Pró-Saúde Centro de Ciências da Saúde e Professor da UFPB.

${ }^{3}$ Enfermeira. Mestranda pelo Programa de Pós-Graduação em Enfermagem da UFPB. Membro do GEPSMEC.

${ }^{4}$ Psicológa. Mestranda pelo Programa de Pós-Graduação em Enfermagem da UFPB. Membro do GEPSMEC.

${ }^{5}$ Enfermeira. Doutora em Enfermagem. Professora do Departamento e do Programa de Pós-Graduação em Enfermagem daUFPB. Líder do GEPSMEC.
}

Autor correspondente:

Camilla de Sena Guerra

Universidade Federal da Paraíba

Rua Ant. ${ }^{\circ}$ Assunção de Jesus, 161 - 58052-230 - João Pessoa-PB-Brasil

E-mail: camilla_sena_@hotmail.com
Recebido: 12/09/2012

Aprovado: 03/03/2013 


\section{INTRODUÇÃO}

Com os avanços políticos e sociais ocorrido nas últimas décadas no Brasil, principalmente no tocante às políticas setoriais como saúde e assistência, também se têm percebido mudanças gradativas na área da Saúde Mental, devido à ampliação de práticas inovadoras e inclusivas que reconhecem os novos direitos dos sujeitos, impostos em várias leis brasileiras, fruto das lutas dos movimentos sociais, especificamente o movimento de luta antimanicomial e dos familiares dos usuários.

Nesta perspectiva, a Reforma Psiquiátrica surge de modo a impor ao Sistema Único de Saúde (SUS) uma reconstrução das políticas de assistência direcionadas à saúde mental no Brasil. Este processo iniciou seu percurso na década de 1970, durante a ditadura militar, período no qual a medicalização era o modelo básico de intervenção. O poder centralizador do hospital psiquiátrico e o elevado índice de internações passaram a serem consideradas as causas estruturais das condições desumanas a que eram submetidos os pacientes psiquiátricos ${ }^{(1)}$.

No decorrer da década de 1980 foi intensificado o debate sobre os direitos humanos e sociais, resultando na elaboração da Constituição de 1988, a qual destacou a saúde como essência da vida digna, viabilizando uma Política de Saúde formulada a partir da garantia máxima do direito à saúde ${ }^{(2)}$.

Nessa construção histórica da Saúde Mental brasileira, a normatização e criação dos Centros de Atenção Psicossociais (CAPS), ocorrida ainda na década de 1990, foi impulsionador no desencadeamento de avanços na área. Nesta época, vários segmentos se mobilizaram e passaram a construir experiências de transformação no campo das práticas de assistência em saúde mental em todo o território nacional. Tudo isso, culminando na Lei n. 10.216/01, que dispõe sobre os direitos do portador de transtorno mental, tornando-se um marco na história da Psiquiatria brasileira, por envolver, em tal proposta, profissionais da área de saúde, usuários e familiares ${ }^{(3)}$.

Entretanto, só a partir 2004, o Ministério da Saúde definiu o CAPS como um serviço de saúde e comunitário do SUS e como um lugar de referência e tratamento para pessoas que sofrem com transtornos mentais. A rede CAPS tornou-se uma ampla e moderna cobertura no âmbito da saúde mental no país ${ }^{(3)}$.

Estes Centros são instituições destinadas a acolher pacientes com transtornos mentais, visa estimular a integração social e familiar, apoiá-los em suas iniciativas de busca da autonomia, oferecer-lhes atendimento médico e psicológico. Os referidos Centros possuem como característica principal buscar integrar os usuários a um ambiente social e cultural concreto, designado como seu território, o espaço da cidade onde se desenvolve a vida cotidiana de usuários e familiares. Assim, estes serviços substitutivos constituem a principal estratégia do processo de Reforma Psiquiátrica devendo funcionar como uma rede de cuidado que promova a inclusão social ${ }^{(3)}$.

Durante a trajetória da Reforma Psiquiátrica percebe-se que o desafio dos profissionais dos CAPS é de acolher, acompanhar e incluir as pessoas em situação de sofrimento psíquico, que anteriormente eram excluídas dos espaços públicos e privadas da vida em sociedade. De forma que elas se sintam à vontade para circular livremente na comunidade, reivindicando um dos direitos mais fundamentais do ser humano, discutidos no âmbito do serviço de saúde mental, o direito à liberdade ${ }^{(4)}$.

Nos CAPS as práticas de inclusão social acontecem a partir das diversas atividades terapêuticas oferecidas, como psicoterapias individuais ou em grupos, oficinas terapêuticas, atividades comunitárias, atividades artísticas, orientação e acompanhamento do uso de medicação, atendimento domiciliar e aos familiares ${ }^{(3)}$. Diante da atuação destes serviços, consideramos como práticas de inclusão social toda tentativa de resgatar a cidadania dos seus usuários, seja através do acolhimento, da assistência, do resgate ao convívio social, familiar e na sociedade ou através de ações intersetoriais que busquem a integralidade do cuidado, direcionada a este sujeito.

As novas formas de convivência oferecidas por profissionais da saúde permite a inclusão das pessoas em situação de sofrimento psíquico ao convívio social diário, que em alguns momentos precisam de cuidados efetivos, e em outros são sujeitos capazes de se relacionar entre si, com familiares e com todas as pessoas da sua comunidade. Essa experiência é possível a partir do momento em que os profissionais proporcionam aos sujeitos o resgate da subjetividade individual, através do acolhimento, da escuta que resulta no resgate perdas ocorridas em diversos cenários ${ }^{(5)}$.

A estratégia de atenção à saúde mental, utilizada nos CAPS, é o trabalho em equipes multiprofissionais que estimula os profissionais a utilizarem todo o seu potencial criativo na relação com o usuário, para juntos realizarem a produção do cuidado priorizando a inclusão do sujeito no convívio social ${ }^{(6)}$.

De acordo com estudo recente, um dos pontos a ser debatido nos serviços de saúde mental é a impor- 
tância de constituir práticas voltadas à inclusão social, vislumbrando contemplar um dos eixos propostos pela reabilitação psicossocial: o trabalho com valor social, superando a visão de que a pessoa com transtorno mental é improdutiva e incapaz ${ }^{(7)}$.

Nesse sentido, um aspecto aos quais os profissionais da saúde devem estar atentos é a dimensão sociocultural na qual o sujeito está inserido, visto ser esse o lugar capaz de produzir novas formas de relação humana, onde o respeito às diferenças possam transformar as relações entre sociedade e loucura( ${ }^{(8)}$.

A inclusão social pauta-se nos seguintes princípios: celebração das diferenças, direito de pertencer, valorização da diversidade humana, solidariedade humanitária, igual importância das minorias e na cidadania com qualidade de vida. Inclusão social como um processo pelo qual a sociedade se adapta para poder incluir em seus sistemas sociais gerais, pessoas com necessidades especiais e, simultaneamente, estas se preparam para assumir seus papéis na sociedade. Sendo assim, destaca-se como possíveis práticas de inclusão social nos serviços de saúde mental: a inclusão no mercado de trabalho, esportes, turismo, lazer e recreação, a inclusão nas artes, cultura e religião, a inclusão na educação e nos ambientes físicos ${ }^{(9)}$.

Considerando que os profissionais que atuam nos CAPS têm como função primordial promover práticas de inclusão social no território ou através da articulação da rede de cuidado, este estudo nos remete a um questionamento: Qual é a concepção de inclusão social dos profissionais que atuam na atenção psicossocial?

Sendo assim, este estudo empírico objetivou conhecer a concepção sobre inclusão social dos profissionais que atuam na atenção psicossocial do Município de Campina Grande/PB/Brasil.

\section{MÉTODO}

Trata-se de uma pesquisa empírica, de natureza exploratória e descritiva. Foi realizada na rede extra-hospitalar de serviços de saúde mental do Município de Campina Grande- Paraíba, Brasil. A referida rede é composta por: dois CAPS i, dois CAPS I, um CAPS II, um CAPS III, um CAPS Ad.

No período de coleta do material empírico existiam 200 profissionais de saúde atuando nos serviços extra-hospitalar de saúde mental. No entanto, por tratar-se de um estudo qualitativo, optou-se por uma amostra de 9,5\%, composta por profissionais de nível técnico e superior que atendessem os critérios de inclusão do estudo: 1) estar atuando há pelo menos um ano na rede de cuidado de saúde mental, tal escolha estabeleceu-se por esse ser um tempo suficiente para que o profissional tivesse propriedade para falar da temática; 2) Possuir vínculo empregatício com o serviço; 3) Ser profissional da saúde de nível superior ou médio e 4) Ter disponibilidade para participar da pesquisa.

Deste modo, 19 profissionais foram escolhidos de forma intencional e por acessibilidade. A coleta do material empírico ocorreu no mês de junho a julho de 2010.

Como instrumento de coleta do material empírico, utilizou-se um roteiro de entrevista semiestruturada, contemplando questões relativas às concepções dos profissionais sobre o que eles compreendiam sobre inclusão social. A entrevista foi gravada com ajuda de um aparelho de Media Player 4 (MP4) e, posteriormente, transcrita na íntegra e de forma literal. O material empírico foi analisado através da técnica de análise de conteúdo categorial temática, em que o tema é uma unidade de significação que se desliga, naturalmente, do texto sob análise, segundo a teoria que serve de base para a leitura ${ }^{(10)}$.

Assim, em conformidade com a técnica, procedeu-se à constituição do corpus, através das entrevistas, transcritas com absoluta fidelidade; leitura flutuante e exaustiva dos textos das entrevistas, recortando e realizando uma síntese geral. Isso possibilitou a visualização das ideias centrais, agregação das falas mais relevantes em temas e finalmente, a análise final do material, estabelecendo um movimento dinâmico entre os dados empíricos, organizados em categorias temáticas, com o referencial teórico pertinente ao tema ${ }^{(10)}$.

Assim, levou-se em consideração literaturas que revelassem avanços obtidos com a implantação e consolidação dos princípios do SUS e com a Reforma psiquiátrica, que, por sua vez, apresentassem progressos no processo de inclusão e acessibilidade das pessoas com transtorno mental. Além disso, estabeleceu-se como referencial para a análise, o conceito de inclusão social $^{(9)}$, o qual se encontra descrito na introdução desse artigo.

A pesquisa foi desenvolvida de acordo com os aspectos éticos recomendados pela Resolução 196/96 do Conselho Nacional de Saúde. Por se tratar de um estudo envolvendo seres humanos, a pesquisa foi submetida à apreciação do Comitê de Ética em Pesquisa da Universidade Federal da Paraíba que emitiu parecer favorável protocolo CEP/HULW n. $264 / 10$. 


\section{RESULTADOS}

Fizeram parte deste estudo 19 profissionais da rede extra-hospitalar de saúde mental do município (sete psicólogos, quatro assistentes sociais, dois enfermeiros, um pedagogo, um psicopedagogo, um fisioterapeuta, um arteterapeuta, um técnico em enfermagem, um técnico de informática). Como o estudo resgatou aqueles profissionais que estavam atuando no município por um período de pelo menos um ano, partiu-se do entendimento que estes possuiam algum conhecimento sobre a temática pesquisada. Logo, percebeu-se que embora os entrevistados tivessem profissões diversas, as concepções dos mesmos sobre inclusão social convergiam independente da função desenvolvida, mostrando, com isso, que existe sintonia entre eles e, consequentemente, no serviço.

No material empírico analisado, identificou-se que o termo inclusão social carrega, na concepção dos profissionais, pelo menos dois significados distintos, assim, construiu-se as categorias que se seguem:

\section{Inclusão Social como acesso a bens e serviços}

Os participantes do estudo que abordaram esta concepção podem ter construído tal entendimento a partir do binômio acesso/acessibilidade de modo à incorpará-las ao paradigma da inclusão social. Enquanto outros, ainda transistam no paradigma da reabilitação. Ainda assim, os termos acessibilidade e acesso têm conotações distintas. Nas falas dos profissionais fizemos um recorte para analisar esta concepção de inclusão social como acesso a bens e serviços:

A inclusão social é inserir o cidadão na vida cotidiana, você não ficar à margem [...] é ir ao supermercado, fazer compras, frequentar a escola, isto é está incluído. (P. 02)

A inclusão social para mim é a facilidade, a abertura que estão dando para essas pessoas com alguma dificuldade, algum transtorno, tenham acesso [...] acessibilidade a essa sociedade. (P. 03)

Inclusão social é promover a circulação das crianças e adolescentes que estão aqui em tratamento, em toda a sociedade [...]. É fazer com que eles possam ter experiências de lazer, experiências educacionais, passar por outros serviços de saúde e assim poder circular por vários ambientes, além do CAPS. (P. 18)
Inclusão social é poder vencer o processo [...] fazendo com que a criança, ela participe de outros espaços sociais, da mesma forma que outras pessoas ditas normais [...] que elas possam usufruir desses espaços [...] ter direito, a estarem na escola [...] em eventos sociais onde todos participam e que sejam tratadas da mesma forma, respeitando as diferenças, que todas as pessoas têm [...]. (P. 19)

\section{Inclusão social como reabilitação/reinserção}

O "doente mental" foi e ainda é considerado um peso para a família. A noção de incapacidade para o trabalho permeia o imaginário social, caracterizando o "doente" como uma pessoa inútil. Vencer este preconceito ainda é um desafio para os profissionais e as falas adiante refletem essa concepção:

Inclusão social é a possibilidade que a gente procura de encaminhar os nossos usuários para desenvolver um trabalho produtivo, financeiramente ou não, [...] mas que ele desenvolva também em sua casa, para mim já significa que ele já está sendo incluído. A partir do momento que a gente começa a conquistar essa prática, mesmo no seio familiar então a gente já está começando a entender que nós já estamos trabalhando a inclusão dessa pessoa, a partir da sua própria familia, então vai desde o trabalho que ele desenvolve dentro do seu ambiente familiar até o trabalho que possa lhe garantir uma renda. (P. 14)

[...] inclusão social, é uma maneira que possibilite oportunidade de resgate da vida de nossos usuários, é uma reintegração, é uma forma de mostrar a eles que estão vivos, que são capazes de andar com as próprias pernas [...] retomar a vida deles, de serem considerados normais [...] poderem desfrutar do dia a dia como uma pessoa capacitada, como uma pessoa feliz que pode exercer normalmente suas funções diárias. (P. 16)

Inclusão social é a gente ter a capacidade de oferecer alguma coisa, onde o usuário [...] excluído tanto da familia quanto da sociedade [...] para que ele possa resgatar alguma coisa que ele já teve, ou que ainda não teve [...] onde ele possa sentir prazer [...] sentir-se cidadão com deveres e direitos [...]. (P. 08)

A inclusão social é onde as pessoas podem realmente deixar de ser excluidas, vistas de forma marginalizadas pela sociedade [...] incluir essas pessoas na 
sociedade, que estão à margem, que estão realmente afastadas de algum grupo social, da familia, do trabalho [...] questão religiosa, qualquer tipo de grupo social. Da própria escola [...]. (P. 15)

Conforme observado nos depoimentos, alguns entrevistados fazem referência à pessoa em sofrimento psíquico como um indivíduo que permanece fora da sociedade, mas que necessita ser incluído para poder participar de atividades realizadas por todos os cidadãos. Para os profissionais, os usuários precisam ser resgatados, precisam ser reinseridos no convívio com a comunidade, com os grupos sociais, para que a partir dessas ações sejam reconhecidos como cidadãos.

\section{DISCUSSÃO}

A implantação de serviços comunitários na rede de Saúde Mental e a busca por práticas mais efetivas para garantir a reabilitação e a reinserção social tornou-se um desafio para os profissionais que atuam nos CAPS e em outros serviços comunitários, levando-os a pensarem formas inovadoras de cuidar, evitando a cronificação e o retorno aos hospitais psiquiátricos.

Inseridos no SUS, os CAPS devem está atrelados aos princípios que norteiam os demais serviços de saúde, como acessibilidade, integralidade e resolutividade na atenção prestada. Acolhendo diariamente, com uma equipe interdisciplinar, usuários com transtorno mental grave e seus familiares. Desta forma, tornam-se os dispositivos estratégicos mais eficientes na substituição dos hospitais psiquiátricos ${ }^{(11)}$.

Assim, a acessibilidade possui diferentes significados, sendo considerada como algo adicional à mera presença ou disponibilidade de um recurso, possibilitando a condição de alcance para utilização, com segurança e autonomia, dos serviços de saúde. Enquanto acesso significa ingresso ao sistema de atenção à saúde, indica o grau de facilidade ou dificuldade com que as pessoas obtêm determinados serviços ${ }^{(12)}$.

As ideias visualizadas na categoria 'Inclusão como Acesso' refletem a concepção de inclusão como um fator desafiador na rede de Saúde Mental. Nessa, os profissionais mostram-se provocados a vencer o processo de exclusão, impregnado culturalmente, e que interfere diretamente na efetivação de práticas inclusivas aos usuários.

Para os entrevistados, inclusão social seria promover a livre circulação das pessoas em situação de sofrimento nos espaços sociais, a exemplo do super- mercado e da escola, ou seja, vencer barreiras físicas e cognitivas. Para outros, inclusão é ter acesso ao lazer, à cultura e à educação, vencendo as barreiras da exclusão econômica e cultural. Vale destacar certa ambiguidade nos termos utilizados pelos profissionais, na medida em que associam acesso à acessibilidade criando assim um significante específico para o grupo que compartilha a mesma opinião.

É importante lembrar que uma sociedade para promover qualidade de vida para todos os cidadãos, deve garantir o acesso aos bens e serviços socialmente construídos. Bem como, a acessibilidade aos mesmos de modo a estarem dispostos de forma mais próxima das pessoas viabilizando assim, a livre circulação dos sujeitos nos serviços. O acesso também pode se referir à permanência, usufruto e à qualidade de consumo de um dado serviço e/ou espaço, de modo a garantir a satisfação dos indivíduos ${ }^{(9)}$.

Por conseguinte, alcançar condições de acessibilidade significa conseguir a equiparação de oportunidades em todos os espaços sociais, implica melhorias em novos conceitos, novas posturas e atitudes ${ }^{(13)}$.

Para a efetivação de políticas públicas de inclusão social é necessário a real implementação dos mecanismos que aumentem sua efetividade, eficácia e eficiência, tais como: a formação de capital social; efetiva avaliação das políticas públicas, com consequente utilização dos resultados; implementação de controle sobre os grupos de interesse (lobby); e observância com vistas a exercer controle sobre as interferências decorrentes da globalização ${ }^{(14)}$.

Desta forma, um dos pontos principais para o município avançar na inclusão social será a garantia de acesso e acessibilidade de todas as pessoas portadoras de deficências, sejam elas físicas, ou psiquícas. Para tanto, deve-se investir em campanhas de sensibilização para a sociedade, profissionais e familiares de pessoas em situação de sofrimento psíquico, para que a partir daí rompam os estigmas, barreiras do preconceito e da descriminação. Sobretudo, precisa-se de políticas eficazes que possibilitem esses progressos.

A noção de reabilitação surgiu aos poucos nos CAPS, como um aprendizado e uma conquista natural, e foi tomando forma com estas instituições em vias de implantação e funcionamento, emergindo de acordo com as necessidades e sendo amadurecidas nas práticas cotidianas no interior dos serviços.

O CAPS não é um sinônimo de inclusão social, mas uma estratégia para estimular esse processo ao enfrentar a cultura estigmatizante, ao buscar construir redes 
sociais, ao estimular a autonomia do usuário, ao evitar internações e possibilitar outras formas de $\operatorname{tratar}^{(15)}$.

Sendo um serviço destinado a promover a Reabilitação Psicossocial, tem em seu escopo um grande desafio, que se encontra atrelado a portadores de transtornos mentais severos e persistentes, a exclusão social. Essa é, em essência, um problema de teor político, diretamente vinculada ao aspecto econômico, devido à precarização do emprego, assim como a desagregação familiar e social, que entre esses usuários se torna ainda mais complexo, visto que a via do trabalho é mais delicada em decorrência, sobretudo, dos estigmas atribuídos à doença, acrescido ao fato do desemprego ${ }^{(15)}$.

Diante dessa problemática, no que concerne a assistência, a Reabilitação Psicossocial, enquanto restituição ao acesso dos direitos de cidadania, apresenta-se como um problema, até mesmo para pessoas em condições “normais" de produtividade e competitividade.

Neste contexto, a Reabilitação Psicossocial procura estabelecer melhores negociações entre as necessidades dos pacientes e as oportunidades do contexto. Os seus pressupostos são baseados na construção de uma nova forma de vida autônoma, inserida na sociedade; 0 processo de restituição do poder contratual do usuário, que se desenvolvem em diversos cenários, considerados espaços de troca ${ }^{(16)}$.

Em outro estudo observou-se que existe uma relação entre reabilitação psicossocial e inclusão social pelo trabalho, mostrando que os CAPS desenvolvem atividades cotidianas significativas, contribuindo com a autoestima e incentivando as qualidades pessoais, através das iniciativas de geração de trabalho e renda ${ }^{(17)}$.

As atividades terapêuticas desenvolvidas dentro dos CAPS são democráticas, criativas e estimulam a autonomia dos usuários, possibilitando o poder de decisão e discussão, bem como a construção de sujeitos ativos e produtivos, participantes do processo de construção de sua cidadania.

Assim, os profissionais entrevistados remetem a reabilitação a uma concepção semelhante na qual é entendida como um conjunto de ações que visam a aumentar as habilidades da pessoa, diminuindo o dano causado pelo transtorno mental, e envolve aqueles que fazem parte do processo de saúde-doença, ou seja, usuários, familiares, profissionais e comunidade em geral $^{(18)}$. Para tanto, faz-se essencial que essas pessoas resgatem aquilo que já tinham, ou poderiam vir a ter. Porém, para isso, serão imprescindíveis investimentos em melhores condições de vida que garantam a esses cidadãos os seus direitos.
Contudo, um fator importante a se considerar é que no processo de reabilitação os profissionais estimulem o empoderamento dos usuários, fazendo com que eles resgatem sua capacidade resiliente, enfrentando as condições adversas que permeiam o seu cotidiano. É imprescindível contextualizar a dinâmica da resiliência enquanto objetivo de promover, nos pacientes e nas famílias, uma interessante perspectiva de reabilitação psicossocial, por favorecer a desconstrução de desesperança, tanto pelos pacientes quanto pela equipe de saúde mental.

Os profissionais que atuam na atenção psicossocial devem priorizar a abordagem extramuros, fazendo com que a sociedade também participe do processo de reabilitação do usuário, para que a inclusão deste aconteça de forma natural. Isto significa a mudança do olhar da sociedade para a pessoa doente: de incapaz para capaz, de louco para um indivíduo em sofrimento, de inútil para cidadão. É um processo que envolve construção de autonomia, liberdade e cidadania ${ }^{(19)}$.

Corroborando com esse estudo, uma pesquisa realizada no Rio Grande do Sul identificou que as práticas desenvolvidas pelos profissionais que atuam nos CAPS devem ser voltadas para atingir a integralidade do cuidado. Sobretudo, faz-se fundamental que estas possuam característica do cuidado em liberdade, da promoção da inclusão social e de estratégias intersetoriais, visando superar as adversidades ${ }^{(20)}$.

A reabilitação engloba todos os profissionais, os atores do processo de saúde-doença, ou seja, os usuários, a família dos usuários e a comunidade inteira. Neste sentido, os profissionais da rede de atenção psicossocial de Campina Grande refletem uma concepção reabilitadora na qual se propõem os modelos substitutivos da saúde mental.

\section{CONSIDERAÇÕES FINAIS}

A temática inclusão social na contemporaneidade tornou-se um grande desafio dos pesquisadores, por caracterizar-se como um assunto ainda polêmico, o qual se encontra em fase de consolidação em nosso país. Nesta perspectiva, investigar como os profissionais da rede de cuidado em saúde mental percebem o objeto de suas práticas cotidianas, permite a ampliação do entendimento sobre possíveis estratégias de se garantir a efetivação dos princípios, tanto da Reforma Psiquiátrica quanto do SUS.

De acordo com as concepções dos profissionais acerca da inclusão social, foi possível perceber que estes relacionam e associam o paradigma da reabilitação/ 
reinserção ao da acessibilidade/acesso, como se estes fossem sinônimos. Quando na verdade, um complementa o outro na rede de cuidado em saúde mental, pois no processo de inclusão social é preciso ter a garantia do acesso e acessibilidade aos serviços de saúde e aos mais diversos espaços da sociedade.

Contudo, quando os profissionais fazem mensão à inclusão social como uma possibilidade de acesso/ acessibilidade das pessoas em situação de sofrimento psíquicos nos ambientes sociais e aos bens e serviços, imprimem a necessidade de inclusão em experiências educacionais, lazer e compras, dentre outros. Deste modo, ressaltam como prioridade a efetivação da promoção da cidadania que favoreça a circulação dessas pessoas de forma igualitária na sociedade e nesses ambientes sociais. Demonstrando que, para que essas pessoas sejam incluídas nesses espaços, é preciso que existam ambientes acessíveis e profissionais preparados para promover a inclusão.

Já relacionado à inclusão social como reabilitação/ reinserção os profissionais identificaram a inclusão dos usuários por meio do trabalho, por este desenvolver o aumento da autoestima. Ao passo que este pode ser entendido como fator imprescindível para o processo de reabilitação dos usuários, estímulo ao empoderamento e de poder resiliente individual dos portadores de sofrimento psíquico.

Considerando essa perspectiva, as práticas de inclusão funcionam no sentido de valorizar os relacionamentos afetivos entre usuários, famílias e trabalhadores do CAPS e a rede de serviços. Percebe-se, portanto, que os profissionais participantes da pesquisa atuam na perspectiva da Reforma Psiquiátrica, promovendo saúde mental a partir da inserção social, afastando-se do tradicional modelo curativista representado pelo modelo asilar, o qual tinha como foco somente o indivíduo e a doença.

\section{REFERÊNCIAS}

1. Ferreira G. A reforma psiquiátrica no Brasil: uma análise sócio política. Psicanálise \& Barroco em revista. [Internet] 2006;4(1):77-85 [acesso em 10 nov 2011]. Disponível: http://www.psicanaliseebarroco.pro.br/ revista/revistas/07/REFORMA.pdf

2. Rozani TM, Mota DCB. Política de saúde para a atenção integral a usuário de drogas. In: Prevenção ao uso indevido de drogas: capacitação para conselheiros e lideranças comunitárias. $4^{\mathrm{a}}$. ed. Brasília: Ministério da Justiça; 2011.p. 239-49.
3. Ministério da Saúde (BR). Secretaria de Atenção à Saúde. DAPE. Saúde Mental no SUS: Os Centros de Atenção Psicossocial. Brasília: Ministério da Saúde; 2004.

4. Medeiros AS, Dimenstein M. O serviço residencial terapêutico de Natal na perspectiva de sua equipe técnica. In: Dimenstein M, organizador. Produção do conhecimento agenciamentos e implicação no fazer pesquisa em Psicologia. Natal: EDUFRN; 2009. p. 219-32.

5. Oliveira FB, Fortunato ML, Dantas RM. Residência terapêutica: um espaço de inclusão social. Saúde debate. 2010;34(86):566-75.

6. Ferrari RAP, Thomsom Z, Melchior R. Atenção à saúde dos adolescentes: percepção dos médicos e enfermeiros das equipes da saúde da família. Cad. Saúde Pública. 2006;22(11):2491-5.

7. Leão A, Barros S. Inclusão e exclusão social: as representações sociais dos profissionais de saúde mental. Interface-Comunic., Saude, Educ. 2011;15(36):137-52.

8. Amarante P. A clínica e a reforma psiquiátrica. In: Amarante P. Archivos de saúde mental e atenção psicossocial. Rio de Janeiro: NAU; 2003. p.45-65.

9. Sassaki RK. Inclusão da pessoa com deficiência no mercado de trabalho. São Paulo: PRODEF; 1997.

10. Bardin L. Análise de conteúdo. Lisboa: Editora 70; 2008.

11. Azevedo EB, Ferreira Filha MO, Silva PMC, Silva VCL, Dantas TRS. Práticas intersetoriais que favorecem a integralidade do cuidado nos centros de atenção psicossociais. Rev. Gaúcha Enferm. 2012;33(1):93-9.

12. Vieira EWR. Acesso e utilização dos serviços de saúde de atenção primária em população rural do município de Jequitinhonha, Minas Gerais [dissertação]. Belo Horizonte (MG): Universidade Federal de Minas Gerais; 2010.

13. Ministério da Saúde (BR). A inclusão de pessoas com deficiência no mercado de trabalho. $2^{\mathrm{a}}$ ed. Brasília: MTE, SIT; 2007. [acesso em 10 fev 2012]. Disponível: http://www.acessibilidade.org.br/cartilha_trabalho.pdf

14. Cunha ABO, Vieira-da-Silva LM. Acessibilidade aos serviços de saúde em um município do Estado da Bahia, Brasil, em gestão plena do sistema. Cad. Saúde Pública. 2010;26(4):725-37.

15. Leão B, Barros S. As representações sociais dos 
profissionais de Saúde Mental acerca do modelo de atenção e as possibilidades de inclusão social. Saude soc. 2008;17(1):95-106.

16. Salles MM, Barros $\mathrm{S}$. Vida cotidiana após adoecimento mental: desafio para atenção em saúde mental. Acta Paul. Enferm. 2009;22(1):11-6.

17. Rodrigues RC, Marinho TPC, Amorim P. Reforma psiquiátrica e inclusão social pelo trabalho. Ciênc. saúde. colet. 2010;15(Supl. 1):1615-25.

18. Lussi IAO, Pereira MAO, Pereira Júnior A. A proposta de reabilitação psicossocial de Saraceno: um modelo de auto-organização? Rev. Latino-Am. Enfermagem. 2006;14(3):448-56.

19. Mielke FB, Kantorski LP, Jardim VMR, Olschowsky A, Machado MS. O cuidado em saúde mental no CAPS no entendimento dos profissionais. Ciênc. saúde colet. 2009;14(1):159-64.

20. Pereira DB, Coimbra VCC, Kantorski LP, Oliveira MM, Soares MC, Schrader G. A integralidade no cotidiano das práticas em um centro de atenção psicossocial. Cogitare enferm. 2011;16(3):430-6. 